\title{
From cells, secrets of the secretome leak out
}

Cells talk to each other, and understanding their language could spawn new therapies. "Scientists have known for years that there are intertissue communications, but now we can pick up more of the factors released, such as the hundreds of factors secreted from skeletal muscle," says Matthew Watt, associate professor of physiology at Monash University in Victoria, Australia. Secreted proteins from all cells make up a rich, complex subset of molecules referred to by scientists as the 'secretome'.

The complexity appeared even in early secretome studies. In 2003, Genentech in South San Francisco, California published results from its Secreted Protein Discovery Initiative, which turned up more than 1,000 different proteins secreted by cells (Genome Res. 13, 2265-2270; 2003). Moreover, the Secreted Protein Database website (http:// spd.cbi.pku.edu.cn/)—maintained by the Center for Bioinformatics at Peking University in China-already includes upwards of 18,000 entries of secreted proteins and their sequences.

On 9 June, at the 'Cancer Proteomics 2009' meeting held by the European Molecular Biology Organization (EMBO) in Dublin, Ireland, Richard Simpson discussed the secretome and colorectal cancer. Simpson, who heads the joint proteomics research laboratory at the Ludwig Institute for Cancer Research in Parkville, Australia, and his colleagues use more than 20 cell lines to search for secreted proteins that are specific to colorectal cancer.

Finding the proteins of interest, though, creates an informatics challenge. For one thing, there is always some natural cell death going on in a cell line, and these dying cells release proteins that have nothing to do with the secretome of a particular disease.

A strategy developed by Simpson's team subtracts out those proteins related to natural cell death from the sum of all proteins released by the cell culture. After that subtraction, about 100 proteins remain that Simpson says could be related to this cancer.

Meanwhile, Thomas Conrads of the University of Pittsburgh Cancer Institute in Pennsylvania and his colleagues use a technique in which they label secreted proteins from prostate cancer cell lines with stable isotopes. He says this method has revealed a "large number of unexpected proteins, like nuclear proteins, that are far too abundant to come from a few dying cells."

Beyond cataloging the elements of the secretome, researchers aim to use the

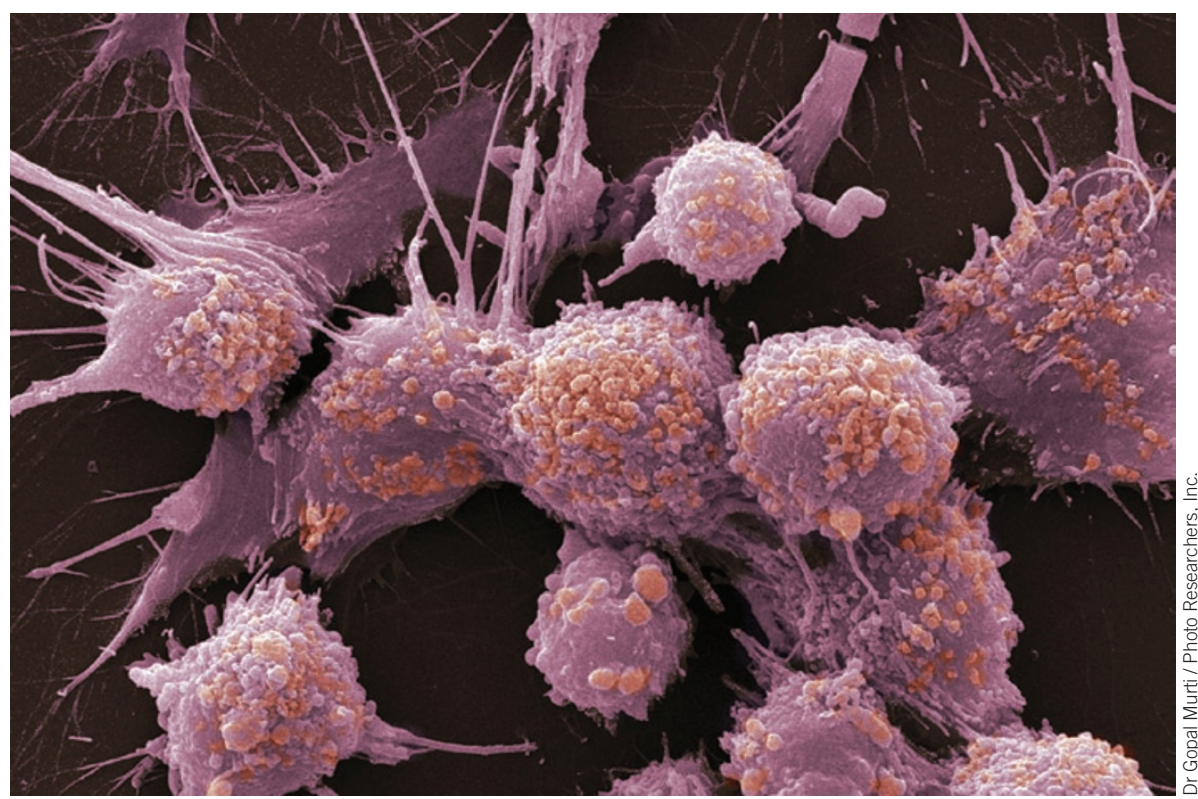

Releasing signals: Studies have identified proteins secreted by prostate cancer cells

secretome to understand disease progression. For example, Christopher Gerner of the Medical University of Vienna in Austria and his colleagues are comparing cells known as fibroblasts from healthy people with fibroblasts in various stages of bone marrow cancer. Their research has shown that, even at intermediate stages of the disease, the secretome includes insulin-like growth factor II, which promotes tumor growth.

Secretome research, though, goes beyond cancer. For example, Watt and his colleagues showed that fat cells in obese mice release more pigment epithelium-derived factor, a protein that can contribute to type 2 diabetes (Cell Metab. 10, 40-47; 2009). In addition, Peter Newman, vice president of research at the Blood Research Institute of the BloodCenter of Wisconsin in Milwaukee, says knowing exactly what platelets secrete into the blood is useful, as these secreted molecules "attract leukocytes, and, over time, this is thought to contribute to the early stages of atherosclerosis."

The cell secretions uncovered by researchers might one day turn into therapies. For example, Jacques Galipeau of McGill University in Montreal found inflammatory cytokines in the secretome of bone marrow stem cells taken from people with Gaucher disease, which leads to skeletal damage. That made Galipeau and his colleagues wonder whether a modified cytokine-one with an added protein - could be engineered to outcompete a secreted cytokine that causes inflammation. To find out, they tested a modified cytokine against mouse models of multiple sclerosis and rheumatoid arthritis. It worked. The modified cytokine reduced the inflammation associated with these diseases, at least in mice. (J. Immunol., 182, 2620ñ2627; 2009; J. Immunol., doi:10.4049/ jimmunol.0900523; 2009). A similar approach might also treat Gaucher and other diseases with an inflammatory component.

As secretome research expands, it will also connect with other projects. For example, pathologist James Versalovic of Baylor College of Medicine in Houston, Texas and his colleagues are sequencing the genomes of specific bacteria as part of the Human Microbiome Project-a US National Institutes of Health effort aimed at characterizing all of the microbes that inhabit humans. In addition, they are studying the secretome of these microbes in the hope of combining the information to develop new treatments for gastrointestinal ailments.

As with other 'omes, however, characterizing the pieces of the secretome is just the first step. Even after identifying biomarkers from a secretome, testing for them in body fluids will require the development of extremely sensitive assays. "The dilution effect from the interstitial tissue to the blood is going to be 1,000 - to 1,500-fold," says Simpson. So it could be some time before information collected about the secretome influences clinical medicine.

Mike May, Houston, Texas 